\title{
Detection of Low Frequency Signals using Interferometric Fiber Sensors based on Phase Generated Carrier Demodulation Technique
}

\author{
Wang Yong-jiao ${ }^{1,2}$, Wang Chuan ${ }^{3}$, Yuan Yin-quan ${ }^{1}$ and Liang Lei ${ }^{1}$ \\ ${ }^{1}$ National Engineering Laboratory for Fiber Optical Sensing Technology \\ Wuhan University of Technology, Wuhan, 430070, China \\ ${ }^{2}$ School of Computer Science, University of Uuban Construction, Pingdingshan, \\ 467036, China \\ ${ }^{3}$ College of Computer and Information Engineering, Henan Normal University, $X$ \\ inxiang, 453007, China
}

\begin{abstract}
A fiber-optic sensor is a sensor with high sensitivity, and signal detection technology is one of its key technologies. Phase generated carrier demodulation technology is a homodyne demodulation method widely used for interference fiber-optic sensors. Research reveals that the amplitude of the interference signal and the modulation depth will affect the result of demodulation. The distortion of the demodulated signal can be reduced by the elimination of the effects of the interference signal amplitude and modulation depth. A phase generated carrier demodulation method for interference fiberoptic sensors is proposed to detect weak ELF signals. Theoretical analysis and simulation have been implemented on the demodulation of weak ELF signals using low-level submixer carrier. The results indicate that in the demodulation of weak ELF signals, DC drift occurs in the DCM approach, while the Arctangent approach does not have this problem. Then, an optical fiber sensing system for ELF signals is established based on optical fiber interferometer, indicating the Arctangent approach is able to demodulate the VLF signals accurately.
\end{abstract}

Keywords: fiber-optic sensors, phase generated carrier demodulation, weak ELF signals

\section{Introduction}

Extremely low frequency signals $(0 \sim 20 \mathrm{~Hz})$ are widely found in nature and human life environment. Earthquakes, tsunamis, volcanic eruptions and other natural phenomena, as well as nuclear explosions, the traveling of ships, the shaking of tall buildings and bridges and other human activities will release a wealth of extremely low frequency signals. Interferometric fiber-optic sensors are applied to the demodulation of extremely low frequency signals due to high sensitivity and good dynamic response characteristics, achieving detection with high resolution and great dynamic range. Thus, this kind of sensors has caused a wide range of research interests.

M. Zumberge, et al., [1] reported a Michelson interferometer-based fiber-optic seismometer, with a detecting frequency range of $0.0001 \sim 15 \mathrm{~Hz}$. In $2007, \mathrm{~N}$. Beverini, et al., [2] realized an ELF sensing system with detection frequency from $0.05 \mathrm{~Hz}$ to tens of $\mathrm{Hz}$ and resolution of 1 taking fiber-optic laser as the sensing element. Lam, et al., [3] realized an ELF sensing system using radio frequency mode-locked laser source technology to reduce noise, and the detection frequency is from $1 \mathrm{~Hz}$ up to several hundred Hz. In 2010, Sato, et al., [4] realized an ELF 
sensing system with the lowest detection frequency of $1 \mathrm{~Hz}$ based on the reference interferometer and phase generated carrier (PGC) demodulation techniques. As can be seen from the researches mentioned above, interferometric fiber-optic sensor technology is a viable solution to achieve he detection of extremely low frequency weak signals, and has a wide range of applications in many areas, such as military, aerospace, automotive, and marine exploration.

When the fiber is subjected to external disturbance, the interferometer outputs a phase-modulated signal, and the sensor signal can be obtained by detecting the change of phase. Because the phase modulation signal of the interferometer is nonlinear with the phase change, plus the effect of temperature drift, pressure fluctuations, and mechanical jitter, random fluctuations in the signal will emerge, namely the so-called phenomena of phase induced signal fade [5].These factors make the signal detection of interferometric fiber-optic sensors be more complex than normal cases. Currently, demodulation methods of the interferometric fiberoptic sensors contain phase generated carrier demodulation method, optical matching differential interferometry method, and differential delay heterodyne method [6]. Since the advantages such as great dynamic range, high sensitivity, good linearity, and high precision, the phase generated carrier demodulation method has become a hot spot of the fiber sensing signal demodulation technology. Currently, the phase generated carrier demodulation method is the most practical demodulation method in the field of fiber-optic sensing interferometer works, and is the most suitable for fiber-optic hydrophone array telemetry and large-scale group signal detection techniques [7-8].

In this paper, the demodulation principles for phase generated carrier used for interferometric fiber-optic sensors have been introduced; the PGC demodulation method of interferometric fiber-optic sensors used for the detection of extremely low frequency signals has been in-depth explored; theoretical analysis and the simulation on the two kinds of phase decimation algorithms in the PGC demodulation method have been implemented; and the comparative experiment of the two algorithms has been carried out.

\section{The Phenomena of Phase Induced Signal Fade}

In the recent 20 years, a wide range of signal detection techniques have been emerged with the development of fiber-optic sensors [9]. In this part, the physical mechanisms of the phase fading phenomenon, as well as a series of anti-phase fading signal detection methods will be introduced.

\subsection{Physical Mechanisms}

Interferometric fiber-optic sensors mainly include Michelson type and MachZehnder type. No matter what kind of structure using in the interferometer, the output signal can be written as

$$
I=I_{0}(1+v \cos \varphi)
$$

Where, $I_{0}$ is the average intensity of the interferometer output; $v$ is the coherence; $\varphi$ is the total phase difference between the two arms of the interferometer, including the initial phase difference $\varphi_{0}$, phase drift caused by sensor signal $\varphi_{s}$, and phase drift caused by noises $\varphi_{n}$, namely $\varphi=\varphi_{0}+\varphi_{s}+\varphi_{n}$. After photoe1-ectric conversion, Equation (1) can be written as:

$$
V=A+B \cos \left(\varphi_{0}+\varphi_{s}+\varphi_{n}\right)
$$


Where, both A and B are constant linear to the input optical power. B is related to the coherence $v$ of the interferometer. Usually, $\varphi_{n}$ is low frequency large signal $\left(\varphi_{n}>\pi\right)$, and $\varphi_{s}$ is high frequency small signal $\left(\varphi_{s}=1\right)$. When a small variety $\Delta \varphi_{s}$ occurs for $\varphi_{s}$, a small variety $\Delta V$ will occur for the output voltage $V$.

$$
\Delta V \approx-B \sin \left(\varphi_{n}+\varphi_{0}\right) \Delta \varphi_{s}
$$

Due to the effect of $\varphi_{n}$, random fluctuations in the signal will emerge; es pecially when $\varphi_{n}+\varphi_{0}=m \pi$, the sensitivity of the system is the poorest, and the signal is completely eliminated, which is called the phase fading phenomenon. To solve this problem, a variety of anti-phase fading signal detection methods have been proposed.

\subsection{Detection Methods Resisting Phase Induced Signal Fade}

Assuming all interferometers takes Mach-Zehnder type, and the entire optical system is polarization-maintaining, and the active devices, such as phase modulator or frequency shifter, only act on the reference arms. The anti-phase fading signal detection methods mainly contain the following types:

1) PGC modem: PGC modem is to eliminate the effect of random drift effect on the sensor signal through the introduction of a phase-modulated signal outside the bandwidth of the detection signal. The phase-modulated signal can be obtained by the addition of a phase modulator or a frequency modulation into the reference arm. The latter one is also known as FM PGC, which is easy to realize the all optical and the digitization of the signal demodulation of the sensor portion, and is a method with wide application. No matter which way is adopted to achieve PGC detection, the carrier must be added in to the phase, so equation (2) becomes:

$$
V(t)=A+B \cos \left(C_{m} \cos \omega_{m} t+\phi\right)
$$

Where, $\omega_{m}$ is the angular frequency of the modulated signal; $C_{m}$ is the largest phase drift caused by the modulated signal; $\varphi=\varphi_{0}+\varphi_{s}+\varphi_{n}$. Expand equation (4) with the first Bessel function, we get:

$$
\begin{aligned}
V(t)=A+B \cos \{[ & {\left[J_{0}\left(C_{m}\right)+2 \sum_{k=1}^{\infty}(-1)^{k} J_{2 k}\left(C_{m}\right)\left[\cos 2 \mathrm{k} \omega_{m}\right] \cos \phi\right.} \\
& -2\left[\sum_{k=1}^{\infty}(-1)^{k} J_{2 k+1}\left(C_{m}\right) \square \cos (2 \mathrm{k}+1) \omega_{m}\right] \sin \phi
\end{aligned}
$$

Where, $J_{2 k}\left(C_{m}\right)$ is the first k-order Bessel function. Multiply equation (5) with $\cos \omega_{m} t$ and $\cos 2 \omega_{m} t$, we get two mutually orthogonal variable $\sin \phi$ and $\cos \phi$. After a series of conversion, we get $B^{2} J_{1}\left(C_{m}\right) J_{2}\left(C_{m}\right) \frac{d \phi}{d t}$, and thus the signal being proportional to the sensor signal $\phi_{s}$.

2) $3 \times 3$ Coupler Interferometer Method: In this method, the output signal includes both $\sin \phi$ and $\cos \phi$, thus the probability of signal complete elimination has been eliminated. The $3 \times 3$ coupler interferometer method includes orthogonal method and the symmetry method. In practical, the symmetry method is more applicable due to its output is more stable than the orthogonal method. After photo- 
electric conversion, the three output signals of the symmetrical $3 \times 3$ coupler interferometer can be written as:

$$
\begin{gathered}
V_{1}=A+B \cos (\phi+2 \pi / 3) \\
V_{2}=A+B \cos \phi \\
V_{3}=A+B \cos (\phi-2 \pi / 3)
\end{gathered}
$$

The average A can be get by the sum of Equations (6), (7) and (8). The three AC signals are obtained by subtracting A respectively. The sensor signal $\phi$ is obtained through high pass filter.

\section{3) Phase tracking detection}

Phase tracking detection is an active phase compensation method, containing DC phase tracking (PTDC) and AC phase tracking (PTAC). Both the two types are to generate a feedback signal using the output signal of the interferometer, and phase compensation is realized by adding the feedback signal into the phase modulator. The working principle of the PTAC method is introduced briefly in the following. Two phase modulators are introduced into the reference arms, so equation (2) becomes

$$
V(t)=A+B \cos \left(\phi-C_{m} \cos \omega_{m} t-\phi_{m}\right)
$$

Where, $\phi_{m}$ is the compensated phase generated by one modulator; $C_{m} \cos \omega_{m} t$ is the compensated phase generated by the other modulator. Set $\phi_{p}=\phi-\phi_{m}$, then equation (9) can be written as:

$$
V(t)=A+B \cos \left(C_{m} \cos \omega_{m} t-\phi_{p}\right)
$$

Multiply equation (10) with $G \cos \omega_{m} t$ and low pass filter, we can get the feedback control signal $G B J_{1}\left(C_{m}\right) \sin \phi$. After lockup, the phase $\phi_{m}$ of the feedback signal is always to counteract $\phi_{p}=1$. The final output signal can be written as $G \gamma \phi$, where $G=G B J_{1}\left(C_{m}\right), \gamma$ is a small value. The sensor signal $\phi$ can be obtained through high pass filter.

\section{The Application of Phase Generated Carrier Demodulation Technique}

Interferometric fiber-optic sensors transduce physical changes into phase changes of optical signals in fibers. In the direct measurement of phase, random phase drift of environmental noise and the DC drift of demodulation circuits will cause serious effects on demodulation results. Through the introduction of a phasemodulated signal outside the bandwidth of the signal to be detected, the detected signal is located in the sideband of the modulated signal. Thus, the external effect is shifted from the detected signal onto the modulated signal.

\subsection{Principle of Phase Generated Carrier Modulation and Demodulation}

In order to achieve the detection of low-frequency signals, scholars have presented many PGC demodulation methods with a variety of different characteristics, among which, the differential cross-multiplication algorithm and arctangent algorithms are two traditional PGC demodulation algorithms. With the development of researches, in order to reduce the sampling frequency, to avoid distortions caused by associated amplitude, and to eliminate light intensity 
disturbance and total harmonic distortion, many experts and scholars have proposed many improved algorithms.

1) Differential cross-multiplication algorithm

The measured signal can be expanded into the superimposition of cosine functions with different frequencies and different initial phase using Fourier transform. So, it can be treated as a cosine signal in the study of PGC demodulation algorithms. The common expression can be written as

$$
V=A_{s} \cos \left(\omega_{s} t+\phi_{s}\right)
$$

Where, $A_{s}$ indicates the amplitude of the signal, $\phi_{s}$ indicates the initial phase of the signal. For differential cross-multiplication algorithm, using formula (11) and (9), considering the input signal phase delay $\phi_{1}$ of the low-pass filter, the two signals after filtering can be written as

$$
\begin{gathered}
S_{1 D}=-B J_{1}(C) \sin \varphi_{s}(t)= \\
\quad-B J_{1}(C) \sin \left[A_{s} \cos \left(\omega_{s} t+\phi_{s}+\phi_{1}\right)\right] \\
S_{2 D}=-B J_{2}(C) \sin \varphi_{s}(t)= \\
-B J_{2}(C) \sin \left[A_{s} \cos \left(\omega_{s} t+\phi_{s}+\phi_{1}\right)\right]
\end{gathered}
$$

Where $J_{1}(C)$ and $J_{2}(C)$ are the first and second order Bessel function respectively. Through differential cross-multiplication for formula (4) and (5), we get

After integration, formula (14) becomes

$$
L_{D}=-B^{2} J_{1}(C) J_{2}(C) A_{s} \omega_{s} \sin \left(\omega_{s} t+\phi_{s}+\phi_{1}\right)
$$

$$
\begin{aligned}
S_{D C M}= & -\int_{0}^{t} B^{2} J_{1}(C) J_{2}(C) A_{s} \omega_{s} \sin \left(\omega_{s} t+\phi_{s}+\phi_{1}\right) d t \\
= & B^{2} J_{1}(C) J_{2}(C) A_{s} \cos \left(\omega_{s} t+\phi_{s}+\phi_{1}\right)- \\
& B^{2} J_{1}(C) J_{2}(C) A_{s} \cos \left(\phi_{s}+\phi_{1}\right)
\end{aligned}
$$

The first term of the formula is the restored measured signal, with phase delay $\phi_{1}$ related to the input signal; the second term is the DC term related to the initial phase of the measured signal and the phase delay of the filter. In the demodulation results, DC drift has been introduced.

First, the impact of the initial phase of the measured signal of the DC item is explored. The initial phase of the signal is determined by the instantaneous phase of the signal when the demodulating system starts collection, which is related to the generation, transmission, and reception of the signal. In the actual measurement process, it is uncontrollable; therefore, it causes random variations for the DC drift in the demodulation results. Second, the impact of the phase delay of the filter on the DC item is examined. In order to avoid phase distortion of the input signal due to low-pass filtering, finite impulse response (FIR) filter with linear phase is selected in the PGC algorithm. For measured signals at the frequency of $f_{s}$, the phase delay generated by FIR low-pass filter is

$$
\phi_{f}=2 \pi f_{s} \frac{N-1}{2 f}
$$

Where, $f$ is the system sampling frequency, $N$ is the order of the low-pass filter. From (16), under a given system sampling frequency, the value of $\phi_{f}$ is determined by the measured signal frequency and the filter order. For the 
integration process of the PGC algorithm, the lower limit of the integration is given by the initial phase of the measured signal and the phase delay of the filter. In this case, random DC drift is introduced into the demodulation results, therefore it is hard to achieve accurate demodulation for extremely low frequency signals using PGC demodulation algorithm.

2) Arctangent algorithm

For arctangent PGC algorithm, after mixing and low-pass filtering, the measured signal can be expressed as

$$
\begin{aligned}
& S_{1 A}=-B J_{1}(C) \sin \left[A_{s} \cos \left(\omega_{s} t+\phi_{s}+\phi_{1}\right)\right] \\
& S_{2 D}=-B J_{2}(C) \sin \left[A_{s} \cos \left(\omega_{s} t+\phi_{s}+\phi_{1}\right)\right]
\end{aligned}
$$

From formula (17) and (18), we get

$$
L_{A}=-\frac{J_{1}(C)}{J_{2}(C)} \tan \left[A_{s}\left(\omega_{s} t+\phi_{s}+\phi_{1}\right)\right]
$$

Then, solve the arctangent for formula (19), let the phase modulation depth $C=2.63 \mathrm{rad}$, so that $J_{1}(C)=J_{2}(C)$. We get

$$
S_{\text {Arc }}=\arctan L_{A}=A_{s} \cos \left(\omega_{s} t+\phi_{s}+\phi_{1}\right)
$$

It can be seen from the comparison between formula (15) and (20), due to the lack of integration in the arctangent algorithm, the demodulation results only contain phase delay rather than DC drifts. Therefore, it is able to achieve accurate demodulation for extremely low frequency signals using the arctangent algorithm.

\subsection{Simulation of Low Frequency Signals}

Using virtual instrument platform, the two kinds of PGC demodulation algorithms are used to implement the demodulation for extremely low frequency signals. Since the measured signal is located in the band below $20 \mathrm{~Hz}$, in the simulation, set the frequency of the PGC modulation carrier be $100 \mathrm{~Hz}$, phase modulation depth $\mathrm{C}=$ $2.56 \mathrm{rad}$, the sampling rate be $1 \mathrm{ks} / \mathrm{s}$, data processing frame length be $100 \mathrm{ks}$.

\section{1) Effects of the Initial Phase on Demodulation Results}

In the simulation, the measured signal is an extremely low frequency cosine single $\varphi_{s}(t)=A_{s} \cos \left(2 \pi f_{s} t+\phi_{s}\right)$, set $A_{s}=0.1, f_{s}=0.05 H z$. The signal is demodulated using the differential cross-multiplication algorithm and arctangent algorithm respectively. First, the effect of the initial phase on the demodulation results of the two algorithms is discussed. On the premise of filter performance, select low order for the low-pass filter $(\mathrm{N}=401)$. According to equation (16), the phase delay $\phi_{f}(t)=\pi / 40$, and it can be ignored. Set the initial phase $\phi_{s}(t)=0, \pi / 6, \pi / 3$, and the simulation results are shown in Figure 1.

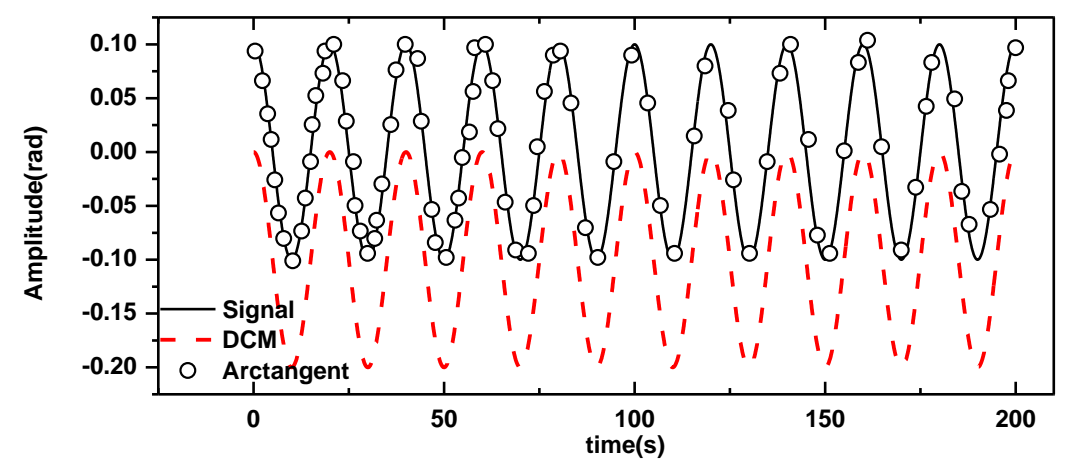



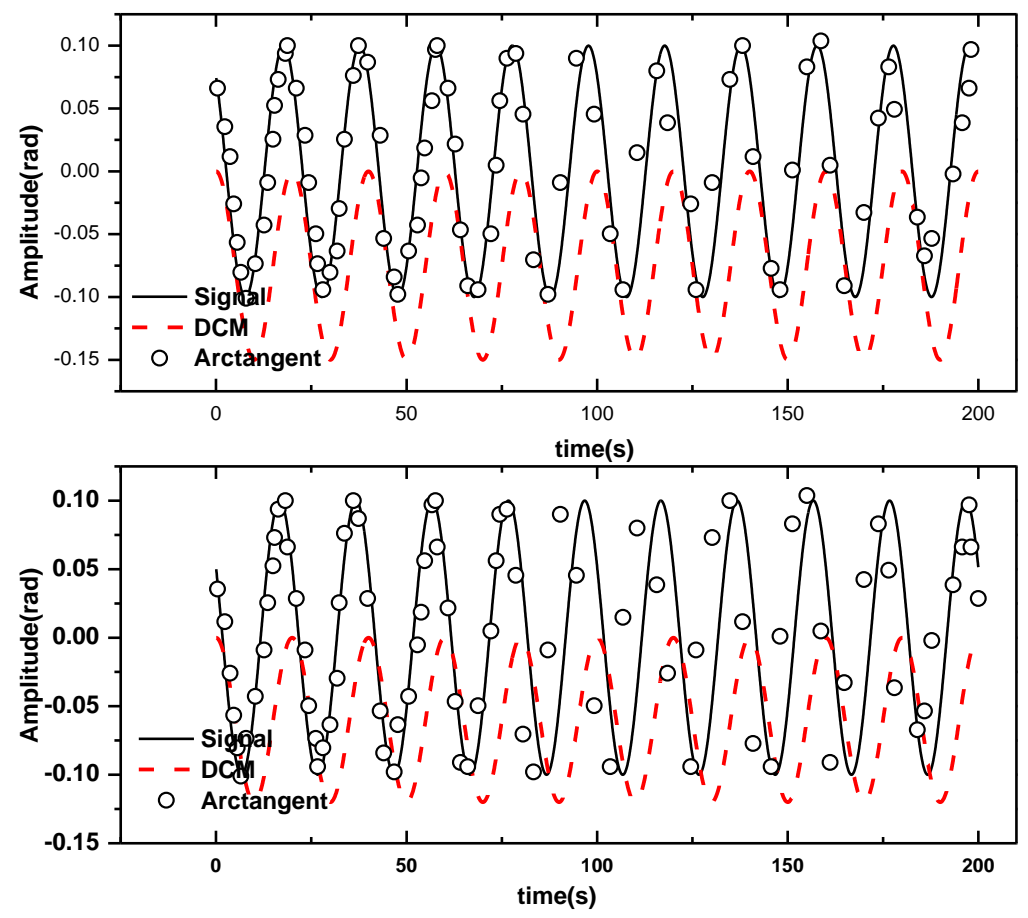

Figure 1. Simulation Results when a) $\phi_{s}(t)=\mathbf{0}$; b) $\left.\phi_{s}(t)=\pi / 6, \mathbf{c}\right) \phi_{s}(t)=\pi / 3$

By the substitution of these simulation parameters into formula (15) and (20), we get the analytical results of the DC drifts of the results of the differential crossmultiplication algorithm and arctangent algorithm. Table 1 lists the analytical results and the simulation results of the two algorithms. Comparing the analytical results and the simulation results, it can be seen that the simulation results are consistent with the analytical results.

Table 1. Analytical Results and the Simulation Results

\begin{tabular}{|l|l|l|l|l|}
\hline Phase & Theoretic(DCM) & Simulation(DCM) & Theoretic(Arc) & Simulation(Arc) \\
\hline 0 & -0.1 & -0.098 & 0 & 0 \\
\hline $30^{\circ}$ & -0.87 & -0.83 & 0 & 0 \\
\hline $60^{\circ}$ & -0.05 & -0.05 & 0 & 0 \\
\hline
\end{tabular}

\subsection{The Effect of Filter Phase Delay on Demodulation Results}

Finite impulse response filter with linear phase is selected as the low pass filter in the system, the upper limit frequency is $50 \mathrm{~Hz}$. Keep $A_{s}=0.1, f_{s}=0.05 \mathrm{~Hz}$, change the filter settings, and set $\mathrm{N}=2001,3334,5001$. According to formula (16), phase delay introduced by the filter $\phi_{s}(t)=0, \pi / 6$, and $\pi / 3$ respectively, simulation results as shown in Figure 2. 

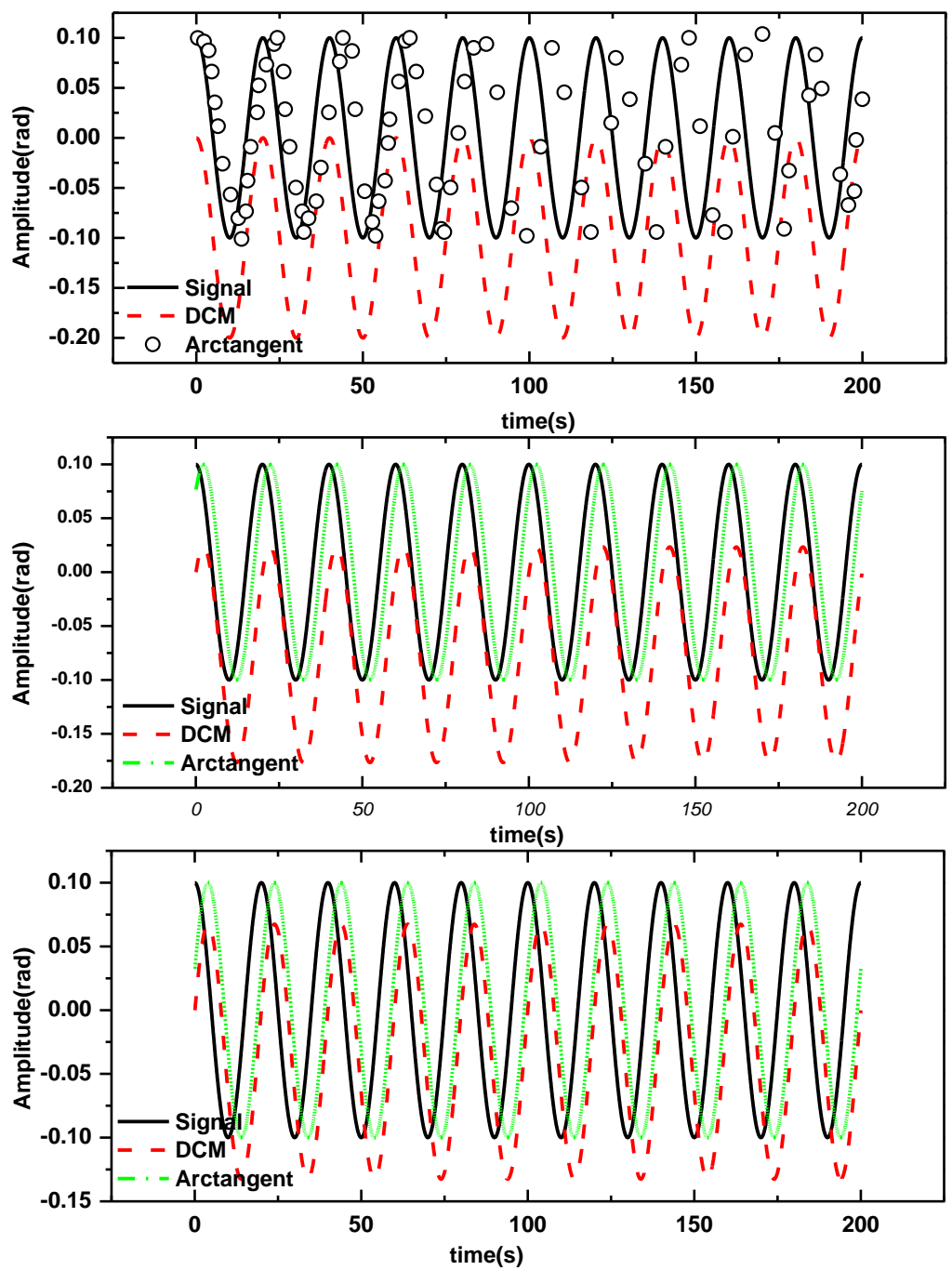

Figure 2. Simulation Results when a) $N=2001$; b) $N=3334$; c) $N=5001$

By the substitution of these simulation parameters into formula (15) and (20), we get the analytical results of the DC term of the results of the differential crossmultiplication algorithm and arctangent algorithm.

Keep FIR filter order number $\mathrm{N}=2001$ unchanged, change the frequency of the input signal, and $f_{s}=0.05 \mathrm{~Hz}, 0.12 \mathrm{~Hz}$. We get the phase delay introduced by the filter: $\phi_{s}(t)=\pi / 10,6 \pi / 25$. The simulation results are shown in Figure 3 .






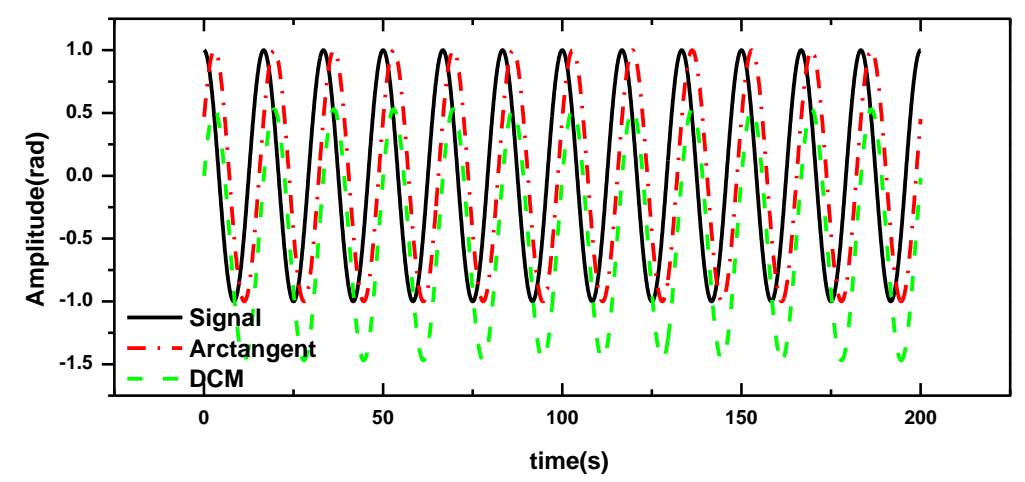

Figure 3. Simulation Results when a) $f_{s}=0.05 \mathrm{H} \mathrm{z}$; b) $f_{s}=0.12 \mathrm{H} \mathrm{z}$

Comparing the analytical results and the simulation results, it can be seen that the simulation results are consistent with the analytical results. In order to realize the detection of weak extremely low frequency signals, an interferometric fiber-optic sensor system is established using the arctangent PGC algorithm, and the performance of this system has been tested.

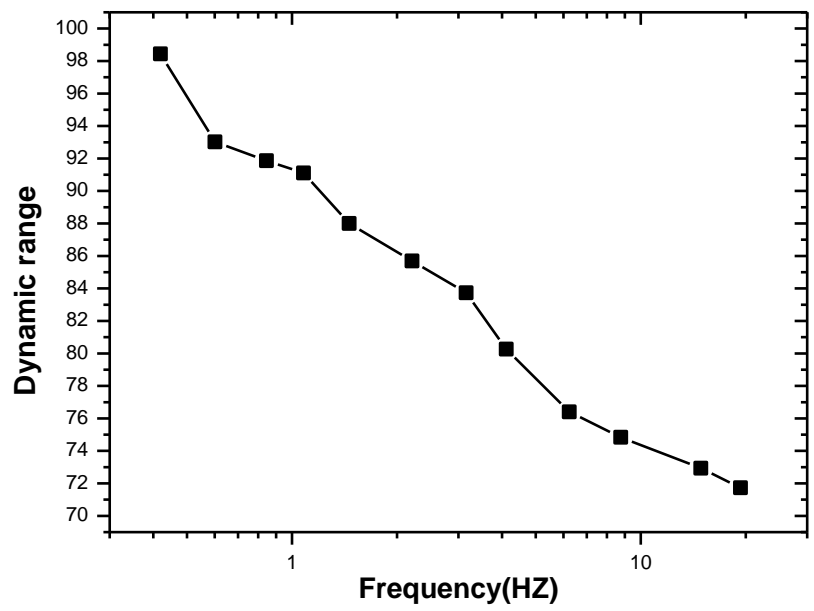

Figure 4. The Dynamic Range of this System

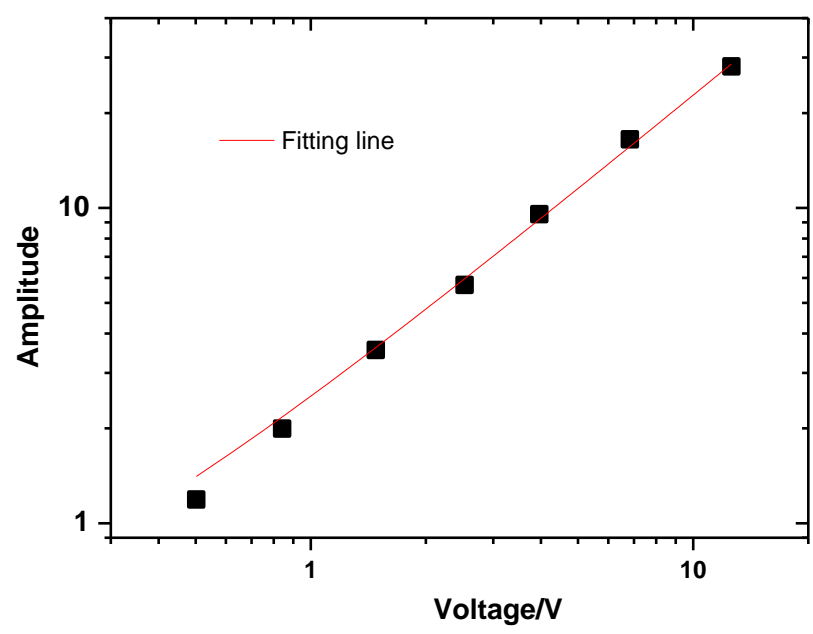

Figure 5. Linear Fitting of the Demodulation Results at $0.1 \mathrm{~Hz}$

The system has achieved the minimum detectable frequency of $0.01 \mathrm{~Hz}$. The minimum detectable signal for the system is $4 \times 10^{-4} \mathrm{rad} / \sqrt{\mathrm{Hz}}$, and the dynamic 
range is shown in Figure 4. At $0.1 \mathrm{~Hz}$, the linear correlation coefficient reaches 99\%, as shown in Figure 5,

\section{Conclusions}

The distortion of demodulated signals can be reduced by eliminating the influence of interference amplitude and modulation depth. Based on this idea, an interferometric fiber-optic sensor phase generated carrier demodulation method is proposed and realized in this paper for the detection of extremely low frequency signals. The selection of low-order mixing carrier is helpful to the reduction of sampling frequency. Theoretical analysis and algorithm simulation on the demodulation of extremely low frequency signals have been carried out. The results show that DC drift occurs in the DCM approach, while the Arctangent approach does not have this problem. Then, an interferometric fiber-optic sensor system is established using the arctangent PGC algorithm, and the performance of this system has been tested. Comparison experiments show that the arctangent algorithm can accurately demodulate extremely low frequency signals, and the results are consistent with the conclusion of theoretical analysis.

\section{References}

[1]. M. A. Zumberge, J. Berger, R. L. Parker and M. Dzieciuch, "U.S. Patent No. 7”, Washington, DC: U.S. Patent and Trademark Office, vol. 224, no. 463, (2007).

[2]. N. Beverini, E. Maccioni, M. Morganti, F. Stefani, R. Falciai and C. Trono, "Fiber laser strain sensor device', Journal of Optics A: Pure and Applied Optics, vol. 9, no. 10, (2007), pp. 958-996.

[3]. T. T. Y. Lam, M. B. Gray, D, A, Shaddock, D. E. McClelland, and J. H. Chow, "Subfrequency noise signal extraction in fiber-optic strain sensors using postprocessing", Optics Letters, vol. 37, no. 11, (2012), pp. 2169-2171.

[4]. K. Sato, H. Kawata, Y. Kashimura and Y. Kamimura, "A measurement of ELF field distributions by using freehand scanning method by Wiimote", In Electromagnetic Compatibility (EMC EUROPE), 2012 International Symposium on, (2012) September 1-4, Rome.

[5]. R. X. Adhikari, (2013), "Gravitational radiation detection with laser interferometry", Rev, Mod. Phys., vol. 86 , no. 121, (2014).

[6]. R. Sambaraju, J. Herrera, J. Marti, D. Zibar, A. Caballero, J. B. Jensen and A. Walber, "Up to 40 Gb/s wireless signal generation and demodulation in $75-110 \mathrm{GHz}$ band using photonic techniques", In Microwave Photonics (MWP), 2010 IEEE Topical Meeting on wave Photonics (MWP), (2010) October 1-4, QC.

[7]. M. Servin, J. C. Estrada and O. Medina, "Fourier transform demodulation of pixelated phase-masked interferograms", Opt. Express, vol. 18, no. 15, (2010), pp. 16090-16095.

[8]. D. Zibar, R. Sambaraju, R. Alemany, A. Caballero, J. Herrera and I. T. Monroy, "Radio-frequency transparent demodulation for broadband hybrid wireless-optical links", Photonics Technology Letters, vol. 22, no. 11, (2010), pp. 784-786.

[9]. F. Focke, D. Schuermann, N. Kuster and P. Schär, "DNA fragmentation in human fibroblasts under extremely low frequency electromagnetic field exposure", Mutation Research/Fundamental and Molecular Mechanisms of Mutagenesis, vol. 683, no. 1, (2010), pp. 74-83.



\section{Author}

Yongjiao Wang, an associate professor in School of Computer Science, University of Uuban Construction, Pingdingshan, city, P.R. China. Her main research area focused on the Optical fiber sensor and image processing. 